\title{
Further Development of a Laser Interferometer
}

\author{
László Rónai \\ Robert Bosch Department of Mechatronics \\ University of Miskolc \\ Miskolc, Hungary \\ ronai.laszlo@uni-miskolc.hu
}

\begin{abstract}
A more than 25 year old laser interferometer has been further developed in order to handle the signals also by computers. A TTL signal converter has been built in using an analog comparator unit type LM319. A National Instruments 6341 OEM measurement card was implemented too. The modernized laser interferometer has been verified with a precise Renishaw XL-80 interferometer. A vibrometer unit has been also calibrated with the updated interferometer.
\end{abstract}

Keywords—laser interferometer; measurement; vibrometer; analog comparator

\section{INTRODUCTION}

The history of the lasers is relatively short, the first laser was made in 1960, which was a solid (ruby) laser. The theoretical basis of the lasers was invented earlier by Albert Einstein [7]. The term of laser [2], [3], [7] is derived from the Light Amplification by Stimulated Emission of Radiation words initial letter. Nowadays laser systems are used in a very wide field, e.g., laboratory researches, measurements, material processing. One can read further information about lasers, e.g., in [2] and [8].

In this paper further development of a laser interferometric motion analyser (LIMA) is discussed. The aim of the development is to handle the signals also by computers. Furthermore the unit will be used in demonstration and educational purposes. In this article comparative measurements are performed with the investigated device and a so called Laser Doppler Vibrometer [9] (LDV) and a Renishaw XL-80 [12] laser interferometer. This research was accomplished at the Institute of Physics [4] in collaboration with the Wigner Research Centre for Physics. The Renishaw interferometer was provided by the Robert Bosch Department of Mechatronics. The precision measurements of vibrations are provided by the laser interferometry.

Three different laser systems are described in Section 2. The designing steps of the further development, e.g., front panel, analog comparator circuit are given in Section 3. The manufacturing steps are written in Section 4. The last two Sections are dealt with the testing process and the practical vibration measurements. The future plans are mentioned in Conclusion.

\section{LASER SYSTEMS}

The application of lasers are relatively popular. The laser beams are highly monochromatic and spatially coherent. Furthermore lasers provide high spectral power density (the energy is concentrated into one point) and little divergence.

The first interferometer was created by Albert Abraham Michelson in 1881, to measure the speed of the Earth compared to the 'ether' in 1887.

The optomechanical scheme of the investigated laser interferometer is described in Fig. 1.

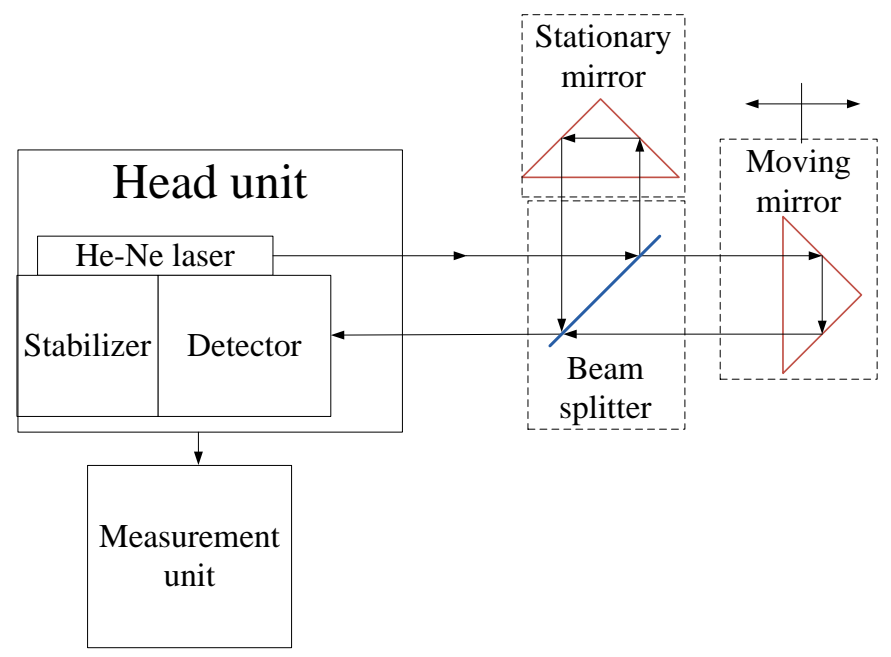

Fig. 1. Optomechanical scheme of LIMA

The system is more than 20 year old, which has got a He$\mathrm{Ne}$ gas laser. The emitted laser beam is passing through a beam splitter, thus the beam is divided into two perpendicular part. The intensity of the two beams are remaining the same [1]. Thereafter one beam is passing through the stationary mirror and the other beam is passing over a moving mirror. The two laser beams are coming across the splitter unit, then it will return to the detector in the head unit. The phase relationship of the beams are determined by the path length difference [1]. The path length difference must be within the coherence range of the laser unit.

The original measurement and data processing module is outdated, which is shown in Fig. 2. The main specifications of the LIMA are given in Table I. 
TABLE I $\quad$ SPECIFICATIONS OF THE LASER UNIT

\begin{tabular}{|l|c|}
\hline \multicolumn{1}{|c|}{ Name of the parameter } & $\begin{array}{c}\text { Value of the parameter } \\
\text { Laser type }\end{array}$ \\
\hline Coherence length & $3 \mathrm{~m}$ \\
\hline $\begin{array}{l}\text { The temperature of the laser } \\
\text { unit in stabilized state }\end{array}$ & $51^{\circ} \mathrm{C}$ \\
\hline Operating temperature & $15^{\circ} \mathrm{C}-25^{\circ} \mathrm{C}$ \\
\hline Laser frequency & $4.7375 \cdot 10^{14} \mathrm{~Hz}$ \\
\hline
\end{tabular}

The communication between the head unit and the measurement unit is provided by a DB 9 connector.

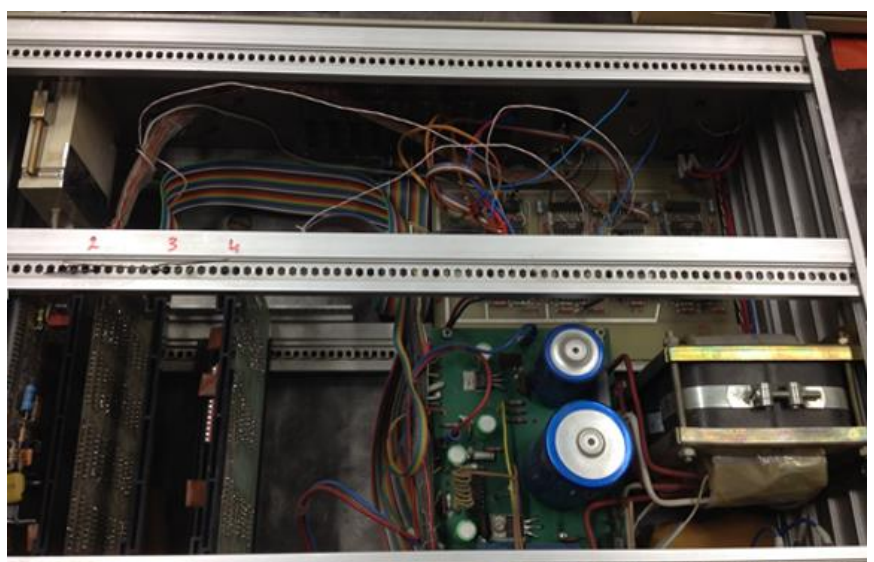

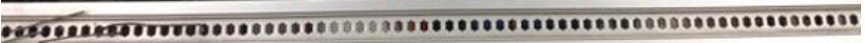

Fig. 2. The original outdated measurement unit

For verification purposes a Renishaw XL-80 type laser interferometer is used. The accuracy of the unit is $\sim 1 \mathrm{~nm}$, the measurement range is expandable to $80 \mathrm{~m}$. The unit communicates with personal computers via an USB cable. This system is applicable for calibration of machine tools, e.g., milling stations, turning machines. The precision of the measurement is ensured by correction units, e.g., humidity, temperature and pressure sensors.

The third laser unit is an LDV, which is a Polytec PDV 100 [10]. The unit is capable for measure the speed and the frequency components of the vibrations with contactless way. The structure of the LDV is compact, thus it is not expandable. The head unit of the system is a He-Ne type gas laser, the wavelength of the beam is $\lambda=632.8 \mathrm{~nm}$, therefore the emitted light is visible. The distance of the measurement is variable within a range of 0.1-30 m. The unit has some main benefits, e.g., the mobility and the simple operation. Easy to analyze objects which are hard to reach. The operation of the system is based on the Doppler Effect. This effect was discovered in 1843 by Christian Andreas Doppler. The essence of the phenomenon is that if the observer and/or the source of the wave move(s) relative to each other, changes of the wave are detected in the wavelengths and the frequencies. The phenomenon can also be observed in astronomy, the red/blue shift relation [11].

\section{DESIGNING PROCESSES}

The designing processes and materials of the measurement system of the LIMA are described in the sequel. Power supply units and clamping elements of the box are selected in accordance with the outdated module and the measured currents.

The Kontakta measurement box type 19" was selected to build the measurement unit. The box had no front panel, thus the designing process has been expanded with planning of the layout of the front panel.

The detector of the head unit sends signals to the measurement box, therefore an analog comparator circuit is designed to provide TTL signals to the measurement card, which is a National Instruments type 6341 OEM card. The above mentioned comparator circuit has been made in the Eagle printed circuit board (PCB) designer software upon the available literature of the old measurement box. The first step is to create the schematic of the circuit, thereafter the PCB model of the board had to be generated in Fig. 3.

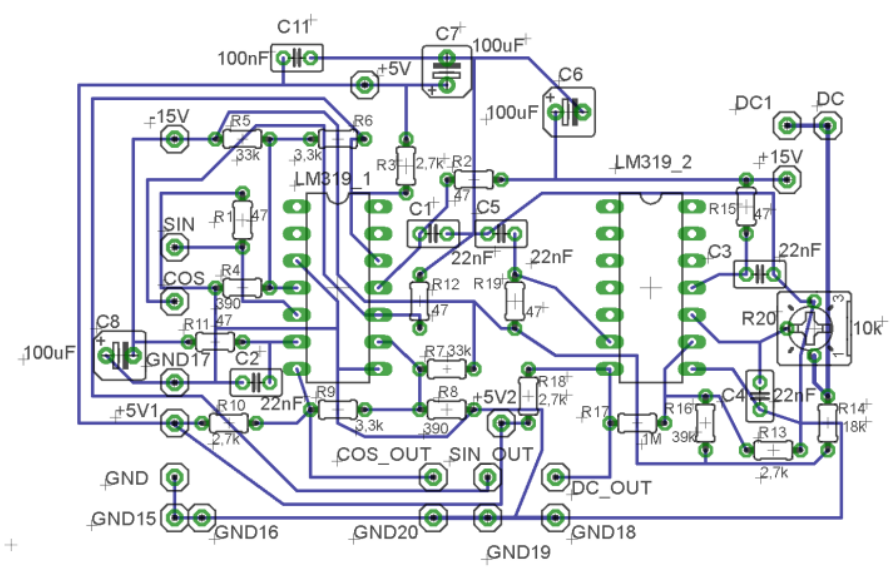

Fig. 3. The generated model of the PCB

The positions of the bores and cutting positions of the front panel are designed in the FrontDesign software. The layout of the bores and the labels are shown in Fig. 4.

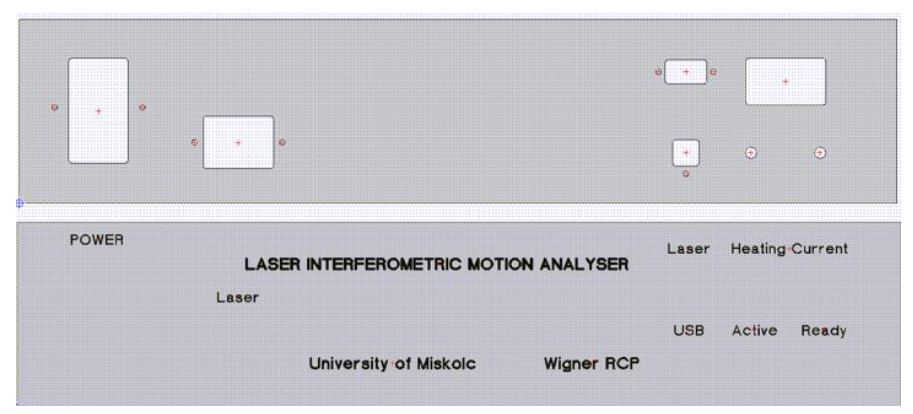

Fig. 4. The layout of the front panel 


\section{THE MANUFACTURING PROCESSES}

The steps of the constructing phases are described in this Section. Firstly the production of the PCB of the analog comparator unit is dealt with. Secondly the milling and the labeling processes of the front panel are discussed.

Performing the milling and the soldering the readymade board without analog comparators is shown in the Fig. 5.

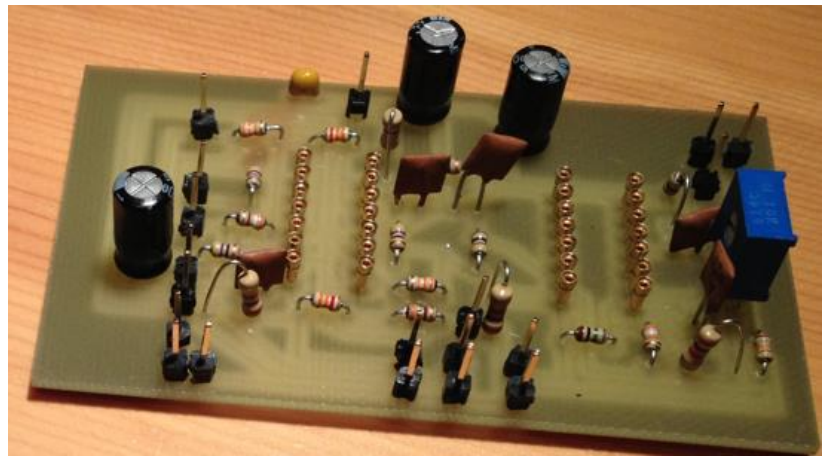

Fig. 5. The readymade PCB

The thickness of the front panel is $4 \mathrm{~mm}$, which consists bores with $\varnothing 3 \mathrm{~mm}$ diameter. The labels of the panel are printed on a transparent adhesive film foil. Thereafter the final assembly and testing of the measurement system have been performed.

\section{THE BUILDING AND TESTING OF THE MEASUREMENT BOX}

At the first step the clamping of the AC connectors, current meter and some other connectors are made. The opportunity of the natural ventilation is an important feature to avoid overheating, thus the distance of the elements are designed relatively large. Spacers are made to fix the subassemblies into the measurement box. To assembly the system some $\varnothing 3 \mathrm{~mm}$ diameter bores had to be drilled then followed by $\varnothing 6 \mathrm{~mm}$ diameter lowering.

The readymade measurement and data processing unit is shown in Fig. 6.

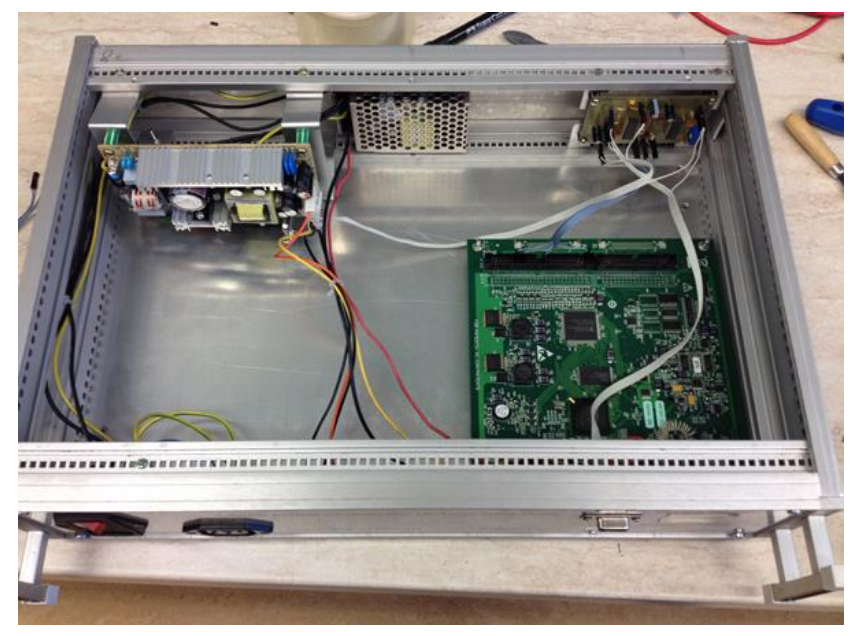

Fig. 6. The modernized measurement system
The further developed system is useable with a PC to perform precision measurements. The data of the measurements are directly evaluated in a PC [5].

\section{PRECISION MEASUREMENT OF VIBRATIONS AND CALIBRATION OF THE LDV UNIT}

The operational accuracy of the further developed measurement unit can be checked by the Renishaw system. The calibration process of the LDV unit has been done by the investigated laser interferometer. The measurements of the displacements are taken place at the same time with the LIMA and the Renishaw system. The arrangement of the measurement is shown in Fig. 7.

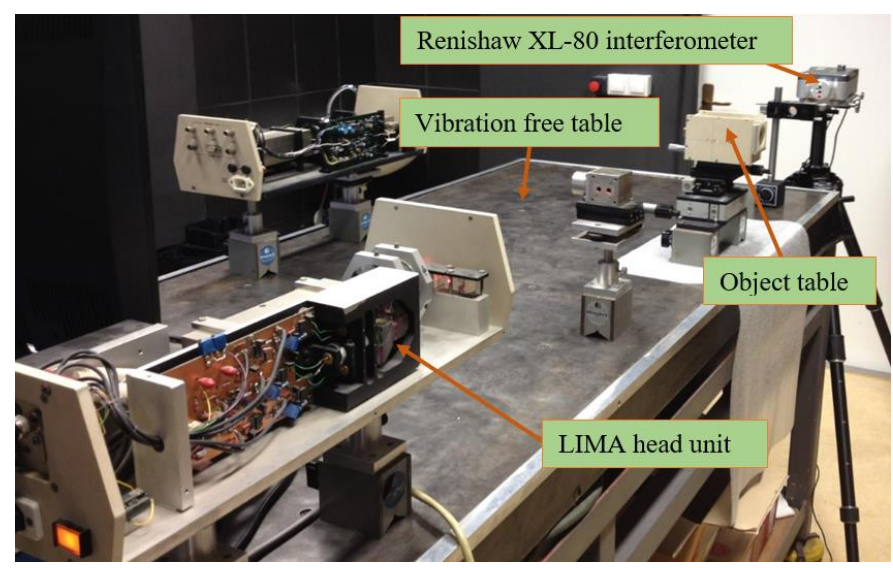

Fig. 7. The arrangement of the displacement measurement

A program had to be written in Scilab software to create the frequency spectrum and the displacement-time diagram of the vibrations of the shocks. The results and the evaluation of the measurements were investigated in detail in the scientific student work [13]. In this paper only some results of the measurement between the investigated interferometer and the Renishaw XL-80 unit are discussed. The results of one of the measurements are shown in Fig. 8. It is important, that the two interferometric systems measure the opposite side of the object table, which is the explanation of the opposite signs obtained for the displacements. Since the reference times of the two measurement units are not harmonized, therefore the time axis is shifted relatively to each other.

The two main frequency components had to be calculated (1) and (2) between the two black lines, which are shown in Fig. 8.

$$
\begin{aligned}
& f_{1, \text { LIMA }}=\frac{2}{0,201 \mathrm{sec}-0,166 \mathrm{sec}}=\underline{\underline{57,143 \mathrm{~Hz}}} \cong f_{1, \text { RENISHAW }}, \\
& f_{2, \text { LIMA }}=\frac{18,5}{0,201 \mathrm{sec}-0,166 \mathrm{sec}}=\underline{\underline{528,57 \mathrm{~Hz}}} \cong f_{2, \text { RENISHAW }} .
\end{aligned}
$$

The velocity data of the LIMA and the LDV unit are different. The velocity results of the LDV are 3.5 times bigger than the calculated results of LIMA [9]. During the calibrating process, the recommended value of the scaling factor, which was given by the manufacturer had to be modified. 


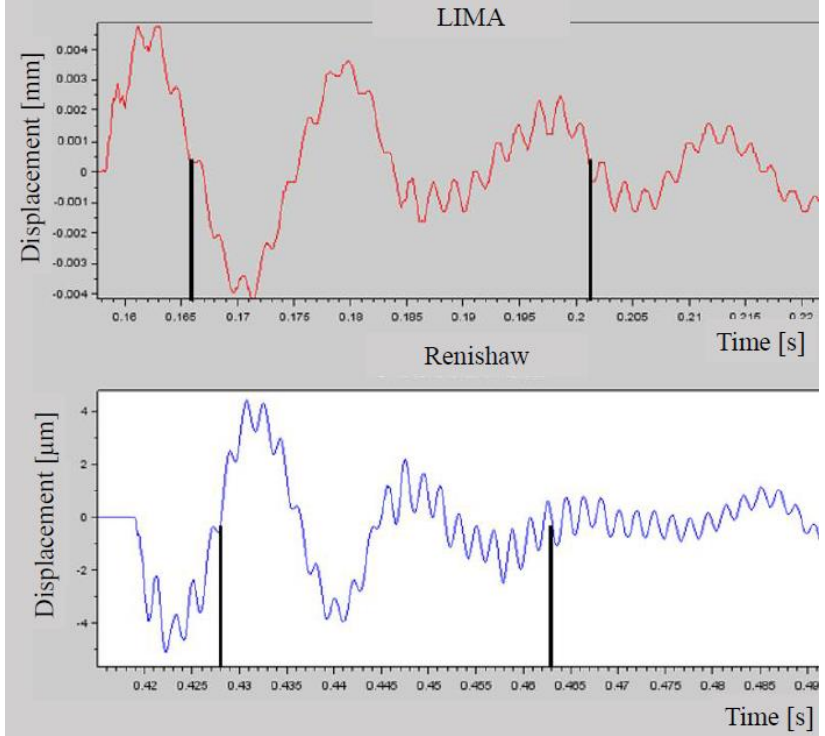

Fig. 8. Partial result of the measurements

\section{CONCLUSION}

In the research this paper was dealing with the development of a laser interferometric measurement and data processing unit, which could be used for vibration measurements and also for demonstrative purposes. Upon the available literature the original measurement unit was reviewed, which helped to design its modernization. During the planning session the schematic of an analog comparator circuit was made and from this a printed circuit board (PCB) was also designed. As the next step the plan of the front panel and its labelling were designed with a software called FrontDesign. Then the position of the bores and cutting positions of the panel were manufactured. The analog comparator circuit was manufactured to convert the signals to TTL.

The comparative precision vibration measurements realized with a Renishaw XL-80 compact interferometer. The LIMA could be perfectly used for carrying out precision displacement measurements up to $\mu \mathrm{m}$ resolution. It was stated that the interferometer and the vibrometer also could show the dominant frequency components. Problems [9] with the calibration of the vibrometer were solved. The calibration was realized with the updated interferometer.

Two modern, operable measuring and data-processing modules were successfully made for the laser interferometer head unit at the Institute of Physics in collaboration with the Wigner Research Centre for Physics.

The development of the software of the unit and the optimization of the beam expansion of the head unit are amongst my plans for the future. Also more precise devices will be used for adjusting the prisms instead of the recently utilized ones.

\section{ACKNOWLEDGMENT}

This research was carried out in the framework of that Center of Excellence of Mechatronics and Logistics at the University of Miskolc.

\section{REFERENCES}

[1] Citrovszky A.: Operating and Maintenance Book of LI-03-type Submicron Laser Interferometer, Budapest 1998 (in Hungarian)

[2] Paripás B.: Lecture of the Laser physics, University of Miskolc (in Hungarian)

[3] Radnóti K.: The Laser, Budapest 1986 (in Hungarian)

[4] Institute of Physics: http://www.uni-miskolc.hu/ www_fiz/

[5] Paripás B., Kovács E., Bodolai T., Tinta T., Rónai L.: Renovation and Development of a Submicron Laser Interferometric Motion Analyser at the University of Miskolc, Report, 2015 (in Hungarian)

[6] JDS Uniphase Corporation - 098 and 1000 series: Gas Laser Catalogue, 2006

[7] Subhash C. S., Haibo Z., Chunlei G., Weiping C.: Nanomaterials: Processing and Characterization with Lasers, John Wiley \& Sons, 2012

[8] Mark Cs.: Funamentals of Light Sources and Lasers, John Wiley \& Sons, 2004

[9] Illavszky V.: Comparation Examination of Vibrations with Laser Ineterferometric Motion Analysers, ME-TDK, 2015 (in Hungarian)

[10] Polytec GmbH: PDV-100 Portable Digital Vibrometer Datasheet, 2012

[11] Jennifer H.: Redshift, University of Exeter, 2009

[12] Renishaw: Brochure of the XL-80 laser measurement system, 2015

[13] Rónai L.: The Development of a Laser Interferometer Measurement System and Precision Analysis of Vibrations, ME-TDK, 2015 (in Hungarian) 